\title{
Registro histórico de Gastrotheca ovifera (Anura: Hemiphractidae): evidencias de disminución en selvas nubladas de la Cordillera de la Costa venezolana
}

\author{
Javier Valera-Leal ${ }^{1 *}$, Aldemar Acevedo $^{2}$, Antonio Pérez-Sánchez ${ }^{2}$, Jorge Vega $^{2} \&$ \\ Jesús Manzanilla ${ }^{1,3}$ \\ 1. Museo del Instituto de Zoología Agrícola (MIZA) Francisco Fernández Yépez, Campus Maracay, Universidad Central \\ de Venezuela, Apartado Postal 4579, Maracay 2101-A, Edo. Aragua-Venezuela; javiervaleraleal@gmail.com \\ 2. Centro de Ecología, Instituto Venezolano de Investigaciones Científicas (IVIC), Apartado 21827, Caracas 1020-A \\ Venezuela; aacevedo@ivic.ve, ajperez@ivic.ve,jvega@ivic.ve \\ 3. Oficina Nacional de Diversidad Biológica, Vice-Ministerio de Conservación, Ministerio del Poder Popular para el \\ Ambiente, Centro Simón Bolívar, Torre Sur, El Silencio, Caracas, Venezuela; jmanzanilla@minamb.gob.ve \\ * Correspondencia
}

Recibido 17-II-2010. Corregido 20-VII-2010. Aceptado 25-VIII-2010.

\begin{abstract}
Historic record of Gastrotheca ovifera (Anura: Hemiphractidae): decline evidence in Venezuelan coastal cloud forests. G. ovifera is a marsupial frog of the cloud and riparian forest from Western and Litoral sections of the Venezuelan Cordillera de la Costa (820-2 000m). This amphibian is considered as an endangered species by the IUCN Species Red List, due to its population decline in pristine and well preserved environments. This conservation status is based on anecdotic interpretations. We collected disperse data from museum records (national and international) and explored the possible association between collection records and precipitation data available for the Henri Pittier National Park (PNHP). Likewise, we carried out a systematic population monitoring of G. ovifera in historic and additional localities among the cloud forest of Rancho Grande, PNHP. We found 106 individuals in 11 zoological collections deposited during 1929-2007. After an effort of 646 hours/ person we did not detect $G$. ovifera individuals in the evaluated localities; as well as no statistical significant associations between the annual precipitation average and the historic records of the species during 1941-1997 period $(r=-0.054, \mathrm{p}=0.820, \mathrm{n}=19)$. We discussed the distribution, fluctuation and population changes of this species, analyzing it conservation status. Rev. Biol. Trop. 59 (1): 329-345. Epub 2011 March 01.
\end{abstract}

Key words: Amphibian decline, Gastrotheca ovifera, Henri Pittier National Park, marsupial frogs, museum data.

Gastrotheca ovifera (Lichtenstein \& Weinland 1854) es una rana marsupial que habita en el dosel arbóreo de bosques nublados y ribereños del Tramo Occidental y Ramal Litoral de La Cordillera de la Costa venezolana (CCV) entre 820-2000m de altitud (Rivero 1961, 1964a, 1964b, Duellman 1980, Duellman et al. 1988, Manzanilla et al. 1995, Barrio 1999, Manzanilla \& Sánchez 2003, Barrio-Amorós 2004, Faivovich et al. 2005). Este anuro se encuentra amenazado bajo la categoría En Peligro (EN) de la Lista Roja de la UICN, debido a que posee una extensión geográfica menor a $5000 \mathrm{~km}^{2}$ y sus poblaciones presentan disminuciones de individuos maduros como consecuencia de alteraciones en su hábitat (La Marca \& Manzanilla 2004). No obstante, esta situación de amenaza no ha sido comprobada por muestreos sistemáticos, sino tan sólo a partir de interpretaciones anecdóticas las cuales sostienen que hasta mediados de la década de 1990 G. ovifera, fue considerada común en hábitats prístinos o ligeramente intervenidos de ambientes de selva nublada del Parque Nacional Henri 
Pittier en la Cordillera de la Costa venezolana (Manzanilla et al. 1995) y que desde entonces sus poblaciones están disminuyendo bruscamente (La Marca \& Manzanilla 2004). Con la finalidad de obtener una mejor aproximación del estatus de conservación de esta especie, recabamos datos dispersos de colección en museos nacionales y extranjeros y exploramos las posibles asociaciones entre los registros del anuro y datos de precipitación disponibles para el PNHP. Complementariamente, realizamos un seguimiento sistemático (monitoreo) de las poblaciones de $G$. ovifera en localidades históricas y adicionales de la selva nublada de Rancho Grande (PNHP) durante 2006 y 2007.

\section{MATERIALES Y MÉTODOS}

Área de estudio: El Parque Nacional Henri Pittier (107 800ha) se encuentra ubicado en el Norte de Venezuela, específicamente al noroeste del estado Aragua dentro de la Cordillera de la Costa venezolana $\left(\mathrm{CCV} ; 10^{\circ} 32^{\prime}-10^{\circ}\right.$ $14^{\prime}$ N, $67^{\circ} 52^{\prime}-67^{\circ} 24^{\prime}$ W) (Fig. 1; Huber 1986, Fernández-Badillo 2000). Esta localidad exhibe un régimen de precipitaciones unimodal, con un máximo en el mes de agosto y mínimo en el mes de febrero (Fernández-Badillo 2000, Genni et al. 2008). Esta compuesta por una alta diversidad de unidades ecológicas distribuidas desde el nivel del mar hasta los $2437 \mathrm{~m}$ en
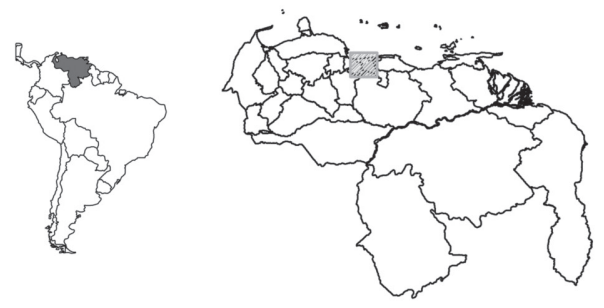

RI (VN)
RII (VN)
RIV (VN)
RIV

PPCP (VS)

$\bigcirc R G(V S)$

स्द्ति QRG(VS)

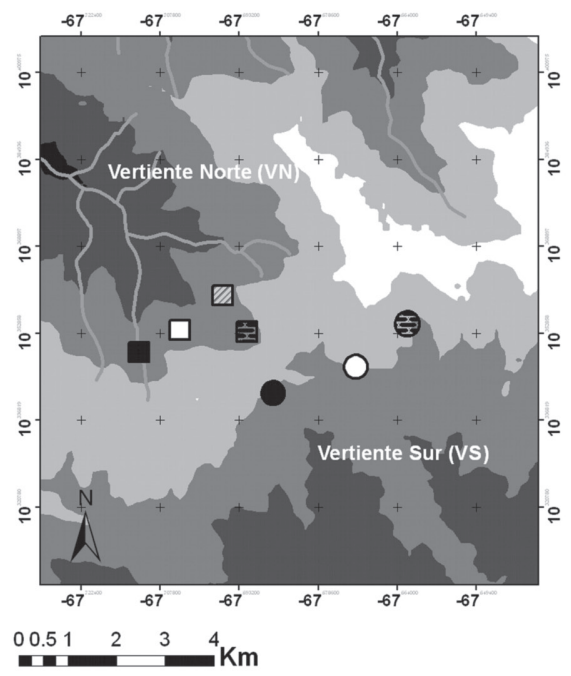

Fig. 1. Ubicación geográfica del área de estudio en el Parque Nacional Henri Pittier, Venezuela. Las localidades históricas y adicionales están representadas por los símbolos en la Vertiente Sur (VS) y Vertiente Norte (VN), respectivamente. Mapa base modificado de Zambrano-Martínez et al. (2004).

Fig. 1. Geographic location of the study area in the Henri Pittier Nacional Park, Venezuela. The historic and additional localities are represented by symbols in the South (VS) and North slope (VN), respectively. The map base was modified from Zambrano-Martínez et al. (2004). 
su vertiente norte y desciende hasta los 490m en su ladera sur (Fig. 1; Huber 1986, 1997, Fernández-Badillo 2000). La composición de anfibios del PNHP es representativa de la CCV y comprende un ensamblaje de 43 especies, de las cuales 11 han sido originalmente descritas a partir de ejemplares de la selva nublada de Rancho Grande y sus alrededores (Valera-Leal et al. 2009).

Registros en museos: compilamos registros históricos a partir de especimenes depositados en cinco museos venezolanos y seis extranjeros (Cuadro 1). Para el primer caso, examinamos ejemplares directamente en cinco colecciones zoológicas de Venezuela. En cuanto a las colecciones foráneas, accedimos a la red internacional de colecciones herpetológicas alojada en el portal electrónico HerpNet 2009. De las 55 instituciones extranjeras consultadas, cinco colecciones de EEUU suministraron información acerca de individuos de G. ovifera depositados. Finalmente, obtuvimos información de Zoologische Staatssammlung

\section{CUADRO 1}

Colecciones zoológicas consultadas y datos recabados para $\mathrm{G}$. ovifera

TABLE 1

Zoological collections consulted and obtained data for G. ovifera

\begin{tabular}{lccc}
\multicolumn{1}{c}{ Colecciones zoológicas } & $\begin{array}{c}\text { Número de } \\
\text { registros }\end{array}$ & Años de colecta & Fuente \\
$\begin{array}{l}\text { Museo de Biología de la Universidad Central de Venezuela } \\
\text { (MBUCV), Caracas-Venezuela. }\end{array}$ & $4(4)$ & $1951,1955,1958,1971$ & Visita y revisión \\
$\begin{array}{l}\text { Museo de Historia Natural La Salle (MHNLS), Caracas- } \\
\text { Venezuela. }\end{array}$ & $20(3)$ & $\begin{array}{c}1950,1955,1956,1966- \\
1971,1974,1980,1984,\end{array}$ & Visita y revisión \\
& & $1987,1994,1996$ &
\end{tabular}

Museo de la Estación Biológica de Rancho Grande,

21 (11) 1964, 1968, 1975-1977, Visita y revisión

Ministerio del Poder Popular para el Ambiente (EBRG), 1980, 1994, 1996, 1997

Maracay-Venezuela.

Museo del Instituto de Zoología Agrícola de la Universidad

Central de Venezuela (MIZA), Maracay-Venezuela.

Carnegie Museum of Natural History (CM), PittsburghEEUU.

Museum of Natural History, University of Kansas (KU), Lawrence-EEUU.

National Museum of Natural History, Division of Amphibian and Reptiles (USNM), Washington D.C.-EEUU.

Texas Cooperative Wildlife Collection (TCWC), Texas

A\&M University, College Station, Texas-EEUU.

University of Illinois Museum of Natural History (UIMNH), Illinois-EEUU.

Zoologische Staatssammlung München (ZSM), MunichAlemania.
1979, 1980, 1985, 1987, 1994, 1996, 2007

$20(2)$

$$
\text { 1929, } 1939
$$

4

1

$106(52)$

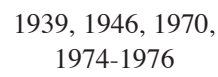

1929, 1965

1980

1939

1929-2007

Los números en paréntesis indican los especimenes recolectados en el Parque Nacional Henri Pittier. The numbers in parenthesis indicate collected specimens in the Henri Pittier National Park. 
München (ZSM, Alemania) mediante la publicación de Glaw \& Franzen (2006).

Datos climáticos: Obtuvimos datos de precipitación registrados en la estación meteorológica 0426 (Rancho Grande-Regresiva) administrada por el Ministerio del Poder Popular para el Ambiente (MINAMB) ubicado a 1 090m de altitud en Rancho Grande-PNHP (10²1'30" N-67\%41'48" W). Seleccionamos datos de lluvia $(\mathrm{mm})$ en un periodo comprendido entre 1941 y 1997 (56 años) con continuidad ligeramente interrumpida (ausencia de registros en abril-junio de 1950, febrero-marzo y julioagosto de 1952, y mayo-junio de 1996). Representamos gráficamente el patrón general de las precipitaciones intra e interanuales, incluyendo una curva de datos suavizados mediante una regresión Lowess (Loess) para el último caso (Cleveland \& Devlin 1988). Calculamos la precipitación media anual, media máxima y mínima anual; así como la media mensual para el conjunto de datos ( \pm desviación estándar). Para determinar posibles asociaciones entre las precipitaciones y el total de número de individuos provenientes del PNHP (inter e intranual), realizamos el cálculo del coeficiente de correlación de rangos de Spearman $\left(r_{s}\right)$ con pruebas de significación (95\% de confianza) a partir de los datos de registros en el periodo antes mencionado; en el anexo 1 se incluyen estos datos. Este coeficiente permite establecer el grado de asociación entre dos variables cuyos datos presentan una distribución desconocida (Quinn \& Keough 2002).

Muestreos: muestreamos durante el periodo 2006-2007, en siete localidades ubicadas en la selva nublada del PNHP entre 840 y $1350 \mathrm{~m}$ de altitud. Dividimos los sitios de muestreos en dos grupos de acuerdo a su ubicación, norte o sur, en cada una de las vertientes del PNHP. El primer conjunto estuvo conformado por las localidades históricas ubicadas en la Vertiente Sur (VS) y correspondieron a Rancho Grande (RG), tramo Paso Portachuelo - Cerro Periquito (PP-CP) y quebrada Rancho Grande (QRG). Por su parte, el segundo conjunto estuvo compuesto por cuatro quebradas tributarias al río La Trilla (Los Riítos de Pittier I, II, III y IV) y fueron incluidas dentro del grupo Vertiente Norte (VN) (Fig. 1, Cuadro 2) (FernándezBadillo 2000). Realizamos todos los muestreos en ambientes de selva nublada de hábitats terrestres o cuerpos de agua, donde establecimos una línea de muestreo de 250-400m de largo por $5 \mathrm{~m}$ de ancho para hábitats terrestres y de ancho variable en quebradas. Todos los

CUADRO 2

Características de las localidades muestreadas

TABLE 2

Features of sampled localities

\begin{tabular}{lcccc}
\multicolumn{1}{c}{ Localidad } & Tipo de hábitat & $\begin{array}{c}\text { Altitud } \\
(\mathrm{m})\end{array}$ & $\begin{array}{c}\text { Longitud del área } \\
\text { de muestreo }(\mathrm{m})\end{array}$ & Ubicación (UTM) \\
RG (VS)* & Terrestre & $1200-1300$ & 300 & $644260 / 1144669$ \\
PPCP (VS)* & Terrestre & $1240-1350$ & 400 & $643881 / 1144712$ \\
QRG (VS)* & Acuático-Terrestre & $1130-1210$ & 396 & $644699 / 1144836$ \\
R I (VN) & Acuático & $1020-1200$ & 254 & $643620 / 1144960$ \\
R II (VN) & Acuático & $980-1120$ & 277 & $643617 / 1144972$ \\
R III (VN) & Acuático & $890-1215$ & 311 & $643580 / 1144283$ \\
R IV (VN) & Acuático & $840-1215$ & 330 & $641013 / 1144241$
\end{tabular}

La información y nombre completo de cada localidad aparece en el texto. Las localidades denotadas por un asterisco (*) corresponden a localidades de recolectas históricas de la especie. The information and complete name of each locality appears in the text. The localities denoted by an asterik $\left(^{*}\right)$ represents the historic sites of the species collect. 
recorridos se realizaron con tiempo restringido entre las 18:00 y 22:00 horas. En cada línea, se estableció detectar individuos de la especie a través de cantos y/o encuentros visuales (sensu Heyer et al. 2001, Lips et al. 2001, Angulo 2006). La intensidad de estos recorridos varió de acuerdo a las condiciones espaciales y temporales presentes en cada una de las localidades seleccionadas (e.g. relieve, accesibilidad, estado de las condiciones hidrometeorológicas).

En cada una de las localidades realizamos entre 6 y 24 muestreos durante enero 2006 y diciembre 2007 (Cuadro 3). Adicionalmente, evaluamos las condiciones naturales del hábitat siguiendo la escala artificial diseñada por Manzanilla \& La Marca (2004) (Cuadro 3).

\section{RESULTADOS}

Datos de Museo: Registramos un total de 106 especimenes de G. ovifera depositados en 11 museos durante el periodo de 1929-2007 (Cuadro 1). Los especimenes mostraron una mayor distribución en el Tramo Occidental y Tramo Central entre 890 y 2 060m de altitud $(\mathrm{n}=105$; Apéndice I). Adicionalmente, reportamos un registro aislado al extremo oriental de Venezuela en el Delta del Orinoco (MHNLS 2942).

Con relación a este registro histórico, 1929 correspondió al año con la mayor cifra de individuos $(17.92 \%, \mathrm{n}=19)$ capturados. En la década de 1970, ocurrió el mayor registro de la especie $(30.18 \%, \mathrm{n}=32)$ a diferencia del periodo 2000-2009 que representó el menor número de recolecciones $(3.77 \%, \mathrm{n}=4)$. Por otro lado, resaltamos que cerca de la mitad del total del registro histórico de G. ovifera (49.05\%, n=52) es proveniente del PNHP (Apéndice I, Cuadro 1), por lo que en 1976 ocurrió el mayor número de anfibios recolectados $(19.23 \%, \mathrm{n}=10)$, además de que en cuya década también encontramos el mayor registro de individuos $(55.76 \%, \mathrm{n}=29)$. A diferencia de esto último, el menor registro para la especie (1.92\%, n=1) ocurrió en el decenio 1930-1939 (Cuadro 3, Fig. 2a).

Del total del registro histórico del PNHP, el $69.23 \%(n=36)$ correspondió a individuos con datos de localidad año y mes de captura

CUADRO 3

Esfuerzo de captura y niveles de calidad del hábitat en las siete localidades muestreadas

TABLE 3

Capture effort and habitat quality levels of the seven studied localities

\begin{tabular}{|c|c|c|c|c|c|}
\hline \multirow{2}{*}{ Localidad } & \multicolumn{2}{|c|}{ Meses de muestreo } & \multirow{2}{*}{$\begin{array}{c}\text { Muestreos } \\
\text { (horas/hombre) }\end{array}$} & \multirow{2}{*}{$\begin{array}{c}\text { Muestreos por } \\
\text { localidad }\end{array}$} & \multirow{2}{*}{$\begin{array}{c}\text { Nivel de calidad } \\
\text { del hábitat }\end{array}$} \\
\hline & 2006 & 2007 & & & \\
\hline RG & Feb, Mar, Jul, Ago & Feb, Mar, Jul, Sep & 96 & 24 & 1 \\
\hline PPCP & Feb, Mar, Jun, Sep & Feb, Mar, Jun, Sep & 192 & 24 & 1 \\
\hline QRG & Ene, Dic & Ene, Dic & 88 & 24 & 2 \\
\hline R I & Ene, Mar, May, Jul, Sep, Nov & - & 62 & 6 & 2 \\
\hline R II & Ene, Mar, May, Jul, Sep, Nov & - & 66 & 6 & 2 \\
\hline R III & Ene, Mar, May, Jul, Sep, Nov & - & 80 & 6 & 2 \\
\hline R IV & Ene, Mar, May, Jul, Sep, Nov & - & 62 & 6 & 2 \\
\hline & & & Total $=646 \mathrm{~h} / \mathrm{h}$ & & \\
\hline
\end{tabular}

Meses de muestreos: enero (Ene), febrero (Feb), marzo (Mar), mayo (May), junio (Jun), julio (Jul), agosto (Ago), septiembre (Sep), noviembre (Nov) y diciembre (Dic). Sampled months: january (Ene), february (Feb), march (Mar), may (May) june (Jun), july (Jul), august (Ago), september (Sep), november (Nov) y december (Dic).

Los niveles de calidad del hábitat van acorde a la siguiente escala: 1) ambiente prístino; 2) ambiente ligeramente perturbado; 3) ambiente modificado 4) ambiente altamente degradado. The quality habitat levels correspond to the following scale: 1) pristine environment, 2) lightly pristine environment, 3) modified environment and 4) highly degraded environment. 

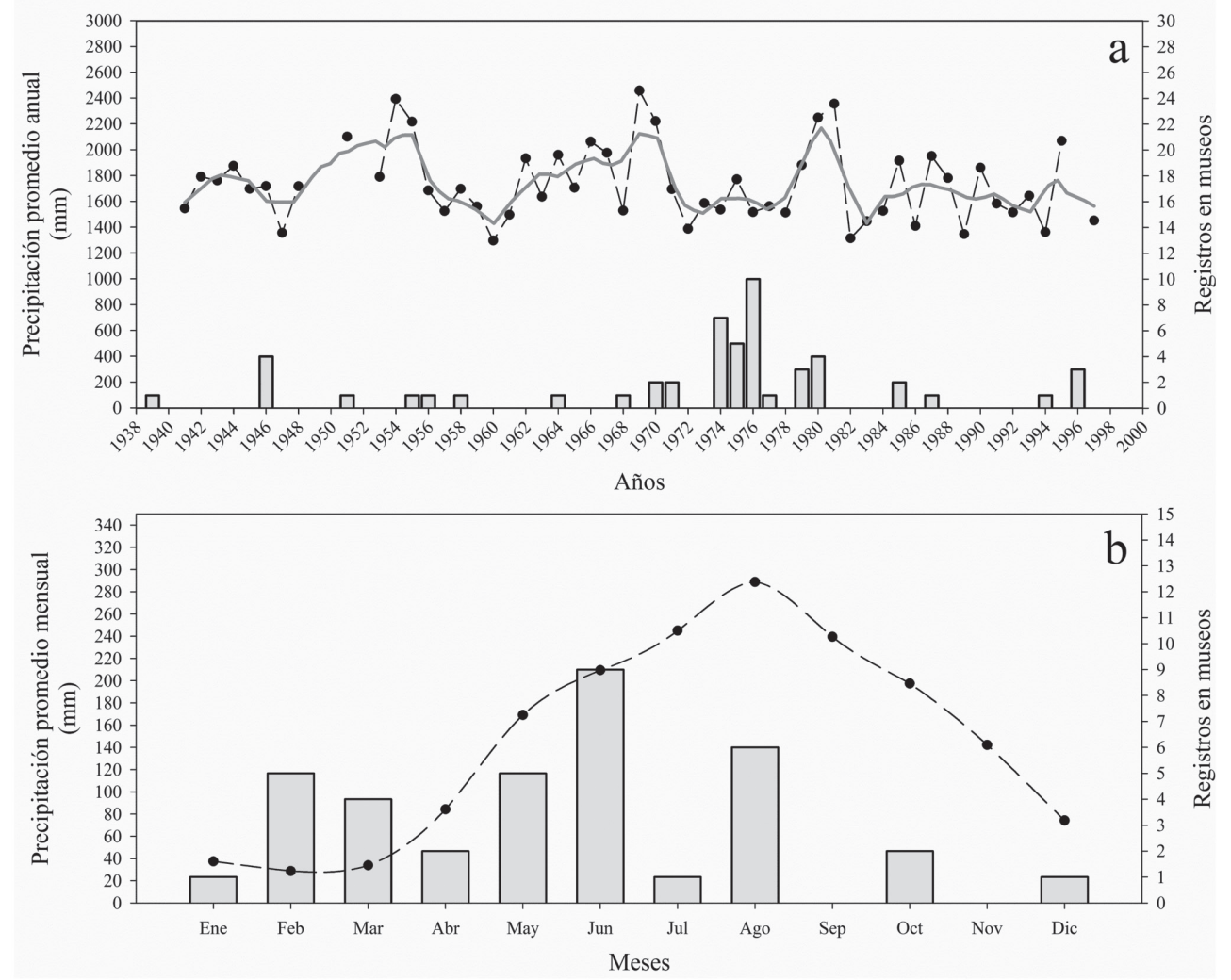

Fig. 2. Distribución temporal de los registros de G. ovifera y patrón de precipitación (inter e intranual) para el Parque Nacional Henri Pittier, Venezuela. a) Registro de especimenes en los 11 museos venezolanos visitados (barras), curva de la precipitación promedio anual observada (puntos y línea discontinua) y curva de precipitación promedio anual suavizada (Loess; línea continua) para el periodo 1941-1997. b) Distribución promedio de los registros históricos de G. ovifera y precipitación promedio mensual en la estación Rancho Grande (PNHP).

Fig. 2. Temporal distribution of G. ovifera records and the pattern of precipitation for the Henri Pittier National Park, Venezuela. a) Specimens records from the 11 museums visited in Venezuela (bars), annual average precipitation curve for both, observed (dots and discontinuous line) and smoothed data (Loess; continuous) during 1941-1997 period. b) Average distribution of G. ovifera records and monthly average precipitation in the Rancho Grande station (HPNP).

que coincidieron con cifras de precipitación. De acuerdo a esta muestra de concurrencia de datos determinamos que junio fue el mes con el mayor número de capturas $(25 \%, \mathrm{n}=9)$ y no se reportaron individuos para septiembre y noviembre (Fig. 2b).

Desde el punto de vista climático, la precipitación media anual fue de $1733.97 \pm 290.08 \mathrm{~mm}$, con lecturas máxima y mínima de $2457.3 \mathrm{~mm}$ en el año 1969 y $1295.9 \mathrm{~mm}$ en 1960, respectivamente (Fig. 2a). De acuerdo al promedio mensual agosto correspondió al mes más lluvioso $(288.8 \pm 76,5 \mathrm{~mm})$, mientras que febrero correspondió al mes más seco $(28.7 \pm 41.7 \mathrm{~mm})$ (Fig. 2b). Dentro del parque, tanto las interpretaciones gráficas como el análisis del coeficiente de Spearman $\left(r_{s}\right)$ no mostraron una asociación estadísticamente significativa entre la precipitación media anual y los registros históricos de la especie por año $\left(r_{s}=-0.054\right.$, $\mathrm{p}=0.820, \mathrm{n}=19$; Fig. 2a); así como tampoco entre la precipitación media mensual y los registros de ejemplares por mes $\left(r_{s}=0.222\right.$, $\mathrm{p}=0.512, \mathrm{n}=10$; Fig. 2b). De igual forma, no 
detectamos ninguna asociación entre el número de ejemplares y los datos mensuales de precipitación en cada evento de captura $\left(r_{s}=0.245\right.$, $\mathrm{p}=0.245, \mathrm{n}=24$ ).

Datos del muestreo: Sobre la base de un esfuerzo total de 646 horas/hombre en las siete localidades seleccionadas en este estudio, no detectamos individuos de G. ovifera en los muestreos de campo durante el 2006 y 2007 (Cuadro 3). Ninguna de estas localidades muestreadas fueron consideradas como hábitats modificados (nivel 3) o degradados (nivel 4) según la clasificación de calidad de hábitats de Manzanilla \& La Marca (2004); siendo predominantes las localidades de calidad de hábitat nivel $2(\mathrm{n}=5$, ambientes ligeramente perturbados) y localidades interpretadas como prístinas ó nivel 1 (RG y PPCP) (Cuadro 3).

\section{DISCUSIÓN}

Distribución geográfica: de acuerdo a nuestros resultados, G. ovifera tiene su mayor distribución en el Tramo Occidental y Ramal Litoral de la Cordillera de la Costa, por lo que consideramos dudoso el reporte señalado para el Delta del Orinoco (ejemplar $\mathrm{N}^{\circ}$ MHNLS 2942, Apéndice I). La fisiografía de esta planicie deltaica y el resto de las localidades señaladas en la Cordillera de la Costa son significativamente contrastantes debido a factores relacionados con la vegetación, altitud, humedad y temperatura (Huber 1997, MARN 2001). Por esta razón, consideramos poco probable que esta especie, predominantemente restringida a un piso climático mesotérmico $\left(12-24^{\circ} \mathrm{C}\right)$ en bosques ombrófilos -nubladosubicados entre 890 y $1950 \mathrm{~m}$, pueda habitar en zonas bajas $(0-200 \mathrm{~m})$ caracterizadas por condiciones macrotérmicas $\left(>24^{\circ} \mathrm{C}\right)$ como las presentes en la bioregión deltaica venezolana.

Fluctuación poblacional: la variación de la abundancia de los anfibios está fuertemente influenciada por las variables ambientales (Duellman 1990, Crump 2001, Scott \& Woodward 2001, Zug et al. 2001, Neckel-Oliveira
2004). Por lo general, es frecuente encontrar las mayores abundancias de anuros tropicales en temporadas lluviosas (McDiarmid 2001). A diferencia de esto, de acuerdo al régimen de precipitaciones presentado en el PNHP, $G$. ovifera no solamente presentó altas abundancias en meses lluviosos de junio (209.38mm, $\mathrm{n}=6$ ) y agosto $(288.75 \mathrm{~mm}, \mathrm{n}=9)$, sino también en febrero $(28.71 \mathrm{~mm}, \mathrm{n}=5)$, que constituye el mes con menor precipitación durante todo el año. Por otro lado, una de las abundancias mas bajas fue registrada en julio $(244.98 \mathrm{~mm}$, $\mathrm{n}=1$ ), mes que forma parte de la temporada de lluvias (Fig. 2). No obstante, es probable que esta información esté sesgada debido a que 1) los datos museológicos no fueron obtenidos homogéneamente desde muestreos sistemáticos; 2) del total de la muestra museológica $(n=52)$ construida a partir de las recolecciones realizadas en el PNHP, sólo logramos estudiar el $69.2 \%(n=36)$, cuyo restante $30.8 \%(n=16)$ careció de información relacionada a datos de precipitación o mes de captura; 3 ) es probable que el número de ejemplares depositados en museos no corresponda a una muestra representativa, debido a que especies del género habitan en el dosel arbóreo del bosque (Manzanilla et al.1995), lo que dificulta su recolección y por ende un bajo éxito de captura. Por tanto, la combinación de estos tres aspectos pudo haber influido en la obtención de una mejor aproximación del patrón poblacional de la especie.

Cambios Poblacionales en el PNHP: desde 1996 hasta el presente, G. ovifera no ha sido registrada en museos, a pesar de que durante el periodo evaluado la especie fue recolectada en 10 de los 12 meses del año. Además, desde principios de la década de 1990, el PNHP ha sido objeto de numerosos inventarios y monitoreos de anfibios (La Marca \& Manzanilla 2004, Manzanilla et al. 1995, Valera-Leal et al. 2009). De acuerdo con estos antecedentes sugerimos que las poblaciones del área de estudio han desaparecido o al menos están presentando un agudo patrón de disminución. 
Esta hipótesis la argumentamos, dado que en nuestra fase de seguimiento poblacional en campo, no registramos ninguna captura y/o detección por canto en localidades seleccionadas, cuyos niveles de calidad de hábitat son prístinos o muy poco intervenidos (Cuadro 3). Esto demuestra un patrón de disminución similar a los ocurridos en otras regiones neotropicales (Bustamante et al. 2005, La Marca et al. 2005, Lips et al. 2005) con localidades sin alteraciones sustanciales del hábitat, lo que sugiere la influencia de otros factores asociados. Con relación a esto, se ha documentado que las enfermedades, cambios climáticos o la sinergia entre estos factores, pudieran estar contribuyendo a la disminución enigmática de los anfibios (Lampo et al. 2006, Stuart et al. 2004). Por ejemplo, el hongo quítrido Batrachochytrium dendrobatidis $-B . d-$ fue recientemente detectado en otras especies del PNHP y en ranas del género Gastrotheca en los Andes venezolanos (Lampo et al. 2008, Nicolás 2007, Rodríguez-Contreras et al. 2008, Sánchez et al. 2008). En el primer caso, individuos de Mannophryne herminae y Atelopus criuciger recolectados en el parque resultaron estar infectados (Nicolás 2007, RodríguezContreras 2008), a lo igual que G. nicefori en la Cordillera andina venezolana (Sánchez et al. 2008). Independientemente de estos resultados, aun se desconoce la existencia y el grado de patogenicidad del hongo en G. ovifera.

Por otra parte, las alteraciones en el régimen climático han sido implicadas en la disminución de anfibios neotropicales (Pounds et al. 1999). Especialmente, en regiones cuyo régimen interanual de precipitaciones ha fluctuado negativamente (Burrowes et al. 2004). El régimen histórico de precipitaciones registrado en la selva nublada de Rancho Grande, tiene una tendencia negativa (Genni et al. 2008), lo que puede traer como consecuencia desbalances fisiológicos -hídricos y renales- (Shoemaker et al. 1992) y desplazamientos poblacionales en masa hacia otras zonas que reúnan condiciones ecológicas más favorables para el cumplimiento del ciclo de vida de la especie (Zug et al. 2001, Dingle \& Drake 2007, Ramenofsky
\& Wingfield 2007). Sin embargo, nuestros resultados de correlaciones no significativas entre las precipitaciones y capturas del anuro obtenidas acá, así como la falta de suministro de información relacionada a otras variables climatológicas (e. g. número de días sin lluvia, humedad relativa y radiación) por parte de la estación meteorológica estudiada, impide determinar con mayor claridad las causas de disminución de la especie en el PNHP.

Para ello, se plantea la necesidad de realizar una evaluación del riesgo asociado a la pérdida de hábitat, enfermedades, cambio climático y el sinergismo entre estos factores, mediante la continuación de seguimientos sistemáticos en el área de estudio y otras localidades del área de distribución de la especie, a fin de obtener una información más precisa sobre el estado de conservación del anuro.

\section{AGRADECIMIENTOS}

Deseamos expresar nuestros agradecimientos a Dinora Sánchez y Francisco Nava por sus comentarios en el escrito. A Dirk Thielen por su colaboración con los datos de precipitación. A Milena Frontado, Mariset Medina, Alejandro Morales, Ronald Luján, Marco Natera y Lenin Reyes por su apoyo en campo. A Carlos Laucho, Damarys Grance, John Lattke y Vilma Savini por permitirnos utilizar las instalaciones de la Estación Biológica de la UCV, Rancho Grande. Así mismo, a Ramón Rivero (EBRG), Fernando Rojas-Runjaic (MHNLS), Javier García y Amelia Díaz de Pascual (CVULA) por el acceso a los datos de las colecciones zoológicas. Este trabajo fue parcialmente financiado por el fondo para la Iniciativa de Especies Amenazadas (IEA), PROVITA, PROYECTO 2009-02.

\section{RESUMEN}

Gastrotheca ovifera es una rana marsupial que habita en el dosel arbóreo de bosques nublados y ribereños del Tramo Occidental y Ramal Litoral de La Cordillera de la Costa venezolana (CCV) entre 820-2 000m de altitud. De acuerdo con la Lista Roja de la UCN se encuentra En 
Peligro (EN) y su evaluación fue realizada de acuerdo a apreciaciones anecdóticas, por lo que su situación de amenaza no ha sido comprobada por muestreos sistemáticos. Con el objetivo de estudiar el estado de las poblaciones de la especie en el Parque Nacional Henri Pittier (PNHP) en el Ramal Litoral de la CCV, realizamos una revisión histórica en museos nacionales y extranjeros y encontramos que la especie fue registrada en 1996 por última vez en el PNHP. Acumulamos 646 horas/persona de muestreo sistemático en localidades históricas y adicionales de la especie en el parque durante los años 2006 y 2007 y no obtuvimos registro de la especie. Adicionalmente, realizamos asociaciones entre datos promedios de precipitación anual y el registro histórico de la especie durante el periodo 1941-1997 ( $r_{s}=-$ $0.054, \mathrm{p}=0.820, \mathrm{n}=19$ ). Discutimos sobre su estado de conservación con base a la distribución, fluctuación y cambios poblacionales de la especie

\section{REFERENCIAS}

Angulo, A. 2006. Fundamentos de bioacústica y aspectos de grabaciones y análisis de cantos, p. 93-104. In A. Angulo, J.V. Rueda-Almonacid, J.V. RodríguezMahecha \& E. La Marca (eds.). Técnicas de Inventario y Monitoreo para los Anfibios de la Región Tropical Andina. Conservación Internacional. Serie Manuales de Campo $N^{\circ}$ 2. Panamericana Formas e Impresos. Bogotá D.C., Colombia.

Barrio, C.L. 1999. Geographic Distribution (Anura): Gastrotheca ovifera. Herpetol. Rev. 30: 106.

Barrio-Amorós, C.L. 2004. Amphibians of Venezuela systematic list, distribution and references, an update. Rev. Ecol. Lat. Am. 9: 01-48.

Burrowes, P.A., R.L. Joglar \& D.E. Green. 2004. Potencial causes for amphibian declines in Puerto Rico. Herpetologica 60: 141-154.

Bustamante, M., S. Ron \& L. Coloma. 2005. Cambios en la diversidad en siete comunidades de anuros en los Andes de Ecuador. Biotropica 37: 180-189.

Cleveland, W.S. \& S.J. Devlin. 1988. Locally-Weighted Regression: An Approach to Regression Analysis by Local. J. Am. Stat. Assoc. 83: 596-610.

Crump, M. 2001. Clima y Ambiente, p. 40-54. In W.R. Heyer, M.A. Donnelly, R.W. McDiarmid, L.C. Hayek \& M.S. Foster (eds.). Medición y Monitoreo de la diversidad biológica, Métodos estandarizados para Anfibios. Editorial Universitaria de La Patagonia, Chubut, Argentina.

Dingle, H. \& A. Drake. 2007. What is migration? Bioscience 57: 113-121.
Duellman, W.E. 1980. A new species of marsupial frog (Hylidae: Gastrotheca) from Venezuela. Occas. Pap. Mus. Zool. Univ. Mich. 690: 1-7.

Duellman, W.E., L.R. Maxson \& C.A. Jesiolowski. 1988. Evolution of marsupial frogs (Hylidae: Hemiphractinae): immunological evidence. Copeia 1988: 527-543.

Duellman, W.E. 1990. Herpetofaunas in neotropical rainforests: comparative composition, history, and resource use, p. 455-505. In A.H. Gentry (ed.). Four Neotropical Rainforests. Yale University, New Haven, Connecticut, USA.

Faivovich, J., C.F.B. Haddad, P.C.A. Garcia, D.R. Frost, J.A. Campbell \& W.C. Wheeler. 2005. Systematic review of the frog family Hylidae, with special reference to Hylinae: Phylogenetic analysis and taxonomic revision. Bull. Am. Mus. Nat. Hist. 294: 1-240.

Fernández-Badillo, A. 2000. El Parque Nacional Henri Pittier. Alcance 60: 1-284.

Glaw, F. \& M. Franzen. 2006. Type catalogue of amphibians in the Zoologische Staatssammlung Munchen. Spixiana 29: 153-192.

Genni, L., E. Degryze \& K. Alvarado. 2008. Análisis de la tendencia y la estacionalidad de la precipitación mensual en Venezuela. Rev. Colomb. Estad. 31: 41-65.

HerpNET. 2009. HerpNET. National Science Foundation, USA. (Consultado 10 marzo 2009, http://www. herpnet.org).

Heyer, W.R., M.A. Donnelly, R. Mc Diarmid, L.C. Hayek \& M.S. Foster (eds.). 2001. Medición y Monitoreo de la Biodiversidad Biológica. Métodos Estandarizados para Anfibios. Editorial Universitaria de La Patagonia, Chubut, República Argentina.

Huber, O. 1986. La Selva nublada de Rancho Grande. Parque Nacional Henri Pittier, El Ambiente Físico, Ecología Vegetal y Anatomía Vegetal. Fondo Editorial Acta Científica Venezolana, Caracas, Venezuela.

Huber, O. 1997. Ambientes Fisiográficos y vegetales de Venezuela, p 279-298. In La Marca, E. (ed.). Vertebrados Actuales y Fósiles de Venezuela. Listados de especies y directorio de colecciones zoológicas, con una introducción a los ambientes fisiográficos y vegetales. Serie "Catálogo Zoológico de Venezuela", Vol. 1. Museo de Ciencia y Tecnología de Mérida, Venezuela.

La Marca, E \& J. Manzanilla. 2004. Gastrotheca ovifera. IUCN Red List of Threatened Species. Version 
2009.1 (Consultado 17 marzo 2009, http://www. iucnredlist.org).

La Marca, E., K.R. Lips, S. Lötters, R. Puschendorf, R. Ibañez, S. Ron, J.V. Rueda-Almonacid, R. Schulte, C. Marty, F. Castro, J. Manzanilla-Pupo, J.E. GarcíaPérez, M.R. Bustamante, L.A. Coloma, A. MerinoViteri, E. Toral, F. Bolaños, G. Chaves, A. Pounds \& B.A. Young. 2005. Catastrophic population declines and extinctions in neotropical harlequin frogs (Bufonidae: Atelopus). Biotropica 37: 190-201.

Lampo, M., A. Rodríguez, E. La Marca \& P. Daszak. 2006. A chytridiomycosis outbreak and a severe dry season precede the disappearance of Atelopus species from the Venezuelan Andes. Herpetol. J. 16: 395-402.

Lampo, M., D. Sánchez, A. Nicolás, M. Márquez, F. NavaGonzález, C.Z. García, M. Rinaldi, F. León, B. Han \& A. Chacón-Ortiz. 2008. Batrachochitrium dendrobatidis in Venezuela. Herpetol. Rev. 39: 449-454.

Lips, K.R., J.K. Reaser, B.E. Young \& R. Ibañez. 2001. Amphibian Monitoring in Latin America: A protocol Manual. Monitoreo de Anfibios en América Latina: Manual de Protocolos. Herpetological Circular No. 30, Society for the Study of Amphibians and Reptiles.

Lips, K.R., P.A. Burrowes, J.R. Mendelson III \& G. ParraOlea. 2005. Amphibian declines in Latin America: widespread population declines, extinctions, and impacts. Biotropica 37: 163-165.

Manzanilla, J., A. Fernández-Badillo, E. La Marca \& R. Visbal. 1995. Fauna del parque Nacional Henri Pittier, Venezuela: Composición y distribución de los anfibios. Acta Cient. Venez. 46: 294-302.

Manzanilla, J. \& D. Sánchez. 2003. Geographic Distribution. (Anura). Gastrotheca ovifera. Herpetol. Rev. 34: 381.

Manzanilla, J. \& E. La Marca. 2004. Museum records and field samplings as sources of data indicating population crashes for Atelopus cruciger, a proposed critically endangered species from the Venezuelan coastal range. Memoria Fundación La Salle de Ciencias Naturales. 157: 5-30.

MARN. 2001. Estrategia nacional sobre diversidad biológica y su plan de acción (eds.). E. Szeplaki, L.B. García-Román, J.C. Rodríguez \& E. González Jiménez. Oficina Nacional de Diversidad Biológica, MARN, Caracas, Venezuela.

McDiarmid, R.W. 2001. Diversidad e Historia Natural de los Anfibios: una síntesis, p. 5-16. In W.R. Heyer, M.A. Donnelly, R.W. McDiarmid, L.C. Hayek \& M.S. Foster (eds.). Medición y Monitoreo de la diversidad biológica. Métodos estandarizados para Anfibios. Editorial Universitaria de La Patagonia, Chubut, Argentina.

Neckel-Oliveira, S. 2004. Effects of landscape on clutches of Phyllomedusa tarsius, a netropicla treefrog. Biol. Conserv. 118: 109-116.

Nicolás, A. 2007. Tendencia altitudinal de infección en un hongo patógeno en ranas Mannophryne herminae del Parque Nacional Henri Pittier. Tesis de Grado, Universidad Simón Bolívar, Caracas, Venezuela.

Pounds, J.A., M.P.L. Fogden \& J.H. Campbell. 1999. Biological response to climate change on a tropival mountain. Nature 398: 611-615.

Quinn, G.P. \& M.J. Keough. 2002. Experimental Design and Data Analysis for Biologists. Cambridge University, Nueva York, EEUU.

Ramenofsky, M. \& J.C. Wingfield. 2007. Regulation of migration. Bioscience 57: 135-143.

Rivero, J.A. 1961. Salientia of Venezuela. Bull. Mus. Comp. Zool. 126: 1-267.

Rivero, J.A. 1964a. The distribution of Venezuelan frogs IV. The Coastal Range. Caribb. J. Sci. 4: 307-317.

Rivero, J.A. 1964b. Salientios (Amphibia) en la colección de la Sociedad de Ciencias Naturales La Salle. Caribb. J. Sci. 4: 297-305.

Rodríguez-Contreras, A., J. Celsa Señaris, M. Lampo \& R. Rivero. 2008. Rediscovery of Atelopus cruciger (Anura: Bufonidae): current status in the Cordillera de La Costa, Venezuela. Oryx 42: 301-304.

Sánchez, D.A., A. Chacón-Ortiz, F. León, B.A. Han \& M. Lampo. 2008. Widespread occurrence of an emerging pathogenic amphibian communities of the Venezuelan Andes. Biol. Conserv. 141: 2898-2905.

Scott, N.J. \& B.D. Woodward. 2001. Relevamientos de lugares de Reproducción, p. 113-120. In W.R. Heyer, M.A. Donnelly, R.W. McDiarmid, L.C. Hayek \& M.S. Foster (eds.). Medición y Monitoreo de la diversidad biológica. Métodos estandarizados para Anfibios. Editorial Universitaria de La Patagonia, Chubut, República Argentina.

Shoemaker, V.H., S.S. Hillman, S.D. Hillyard, D.C. Jackson, L.L. Mcclanahan, P.C. Withers \& M.Y. Wygoda. 1992. Exchange of water, ions and respiratory gases in terrestrial amphibians, p. 125-150. In M.E. Feder \& W.W. Burggren (eds.). Environmental physiology of the amphibians. University of Chicago, Chicago, Illinois, EEUU. 
Valera-Leal, J.A., J. Manzanilla, D. Sánchez \& M. NateraMumaw. 2009. Anfibios del Parque Nacional Henri Pittier y zonas aledañas: distribución, taxonomía, historia natural y estado de conservación, p 349. VIII Congreso Venezolano de Ecología. Libro de resúmenes, Santa Ana de Coro, Falcón, Venezuela.

Zambrano-Martínez, S., R. Lazo, M.A. Oliveira-Miranda \& C. Kalinhoff. 2004. Parque Nacional Henri Pittier. In J.P. Rodríguez, R. Lazo, L.A. Solórzano \& F. Rojas-Suárez (eds.). Cartografía Digital Básica de las Áreas Naturales Protegidas de Venezuela: Parques Nacionales, Monumentos Naturales, Refugios de Fauna, Reservas de Fauna y Reservas de
Biósfera. Versión 1.0, CD ROM y en-línea. Centro Internacional de Ecología Tropical (CIET), Instituto Venezolano de Investigaciones Científicas (IVIC), Conservación Internacional Venezuela, UNESCO y Oficina Nacional de Diversidad Biológica del Ministerio del Ambiente y de los Recursos Naturales (MARN). Caracas, Venezuela (También disponible en línea, http://ecosig.ivic.ve).

Zug, G.R., L.J. Vitt \& J.P. Caldwell. 2001. Population Structure and Dynamics, p. 301-313. In Herpetology. An Introductory Biology of Amphibians and Reptiles. Academic, San Diego, California, EEUU. 


\section{APÉNDICE I}

Lista de especimenes, números de colección y localidades de registros para $\mathrm{G}$. ovifera.

Las altitudes subrayadas fueron estimadas mediante el conocimiento previo de las poblaciones y sus respectivas localidades. ${ }^{(\dagger)}$ Esta cohorte se contó cómo un sólo individuo

APENDIX 1

Specimens list, collection numbers and records localities for G. ovifera.

The altitud data underlined was stimated through previous knowlegde of the populations and it respective localities. ${ }^{(\dagger)}$ This cohort was counted as a unique individual

\begin{tabular}{|c|c|c|c|c|c|}
\hline Colección & $\begin{array}{c}\mathrm{N}^{\mathrm{o}} \\
\text { espécimen }\end{array}$ & Localidad & $\begin{array}{l}\text { Fecha de } \\
\text { colecta }\end{array}$ & $\begin{array}{c}\text { Precipitación } \\
\text { correspondiente al mes de } \\
\text { evento de captura registrada } \\
\text { en el PNHP }(\mathrm{mm})\end{array}$ & $\begin{array}{l}\text { Altitud } \\
\text { (metros) }\end{array}$ \\
\hline $\mathrm{CM}$ & 22787 & Venezuela: Edo. Aragua: Colonia Tovar & 1939 & & sin altitud \\
\hline $\mathrm{CM}$ & 22788 & Venezuela: Edo. Aragua: Colonia Tovar & 1939 & & sin altitud \\
\hline $\mathrm{CM}$ & 61858 & $\begin{array}{l}\text { Venezuela: Edo. Aragua: Parque Nacional } \\
\text { Henri Pittier, Rancho Grande }\end{array}$ & 1976 & Dato no precisado & sin altitud \\
\hline $\mathrm{CM}$ & 61859 & $\begin{array}{l}\text { Venezuela: Edo. Aragua: Parque Nacional } \\
\text { Henri Pittier, Rancho Grande }\end{array}$ & 1976 & Dato no precisado & sin altitud \\
\hline $\mathrm{CM}$ & 7706 & Venezuela: Edo. Aragua: Colonia Tovar & 1929 & & 1800 \\
\hline $\mathrm{CM}$ & 7707 & Venezuela: Edo. Aragua: Colonia Tovar & 1929 & & 1800 \\
\hline $\mathrm{CM}$ & 7708 & Venezuela: Edo. Aragua: Colonia Tovar & 1929 & & 1800 \\
\hline $\mathrm{CM}$ & 7709 & Venezuela: Edo. Aragua: Colonia Tovar & 1929 & & 1800 \\
\hline $\mathrm{CM}$ & 7710 & Venezuela: Edo. Aragua: Colonia Tovar & 1929 & & 1.800 \\
\hline $\mathrm{CM}$ & 7711 & Venezuela: Edo. Aragua: Colonia Tovar & 1929 & & 1800 \\
\hline $\mathrm{CM}$ & 7716 & Venezuela: Edo. Aragua: Colonia Tovar & 1929 & & 1800 \\
\hline $\mathrm{CM}$ & 7721 & Venezuela: Edo. Aragua: Colonia Tovar & 1929 & & 1800 \\
\hline $\mathrm{CM}$ & 7722 & Venezuela: Edo. Aragua: Colonia Tovar & 1929 & & 1800 \\
\hline $\mathrm{CM}$ & 7723 & Venezuela: Edo. Aragua: Colonia Tovar & 1929 & & 1800 \\
\hline $\mathrm{CM}$ & 7724 & Venezuela: Edo. Aragua: Colonia Tovar & 1929 & & 1800 \\
\hline $\mathrm{CM}$ & 7725 & Venezuela: Edo. Aragua: Colonia Tovar & 1929 & & 1800 \\
\hline $\mathrm{CM}$ & 7726 & Venezuela: Edo. Aragua: Colonia Tovar & 1929 & & 1800 \\
\hline $\mathrm{CM}$ & 7727 & Venezuela: Edo. Aragua: Colonia Tovar & 1929 & & 1800 \\
\hline $\mathrm{CM}$ & 7728 & Venezuela: Edo. Aragua: Colonia Tovar & 1929 & & 1800 \\
\hline $\mathrm{CM}$ & 7768 & Venezuela: Edo. Aragua: Colonia Tovar & 1929 & & 1800 \\
\hline EBRG & 46 & $\begin{array}{l}\text { Venezuela: Edo. Aragua: Parque Nacional } \\
\text { Henri Pittier, Rancho Grande }\end{array}$ & May-64 & 132.3 & 1100 \\
\hline EBRG & 47 & $\begin{array}{l}\text { Venezuela: Edo. Aragua: Parque Nacional } \\
\text { Henri Pittier, Rancho Grande }\end{array}$ & Ago-68 & 183.6 & 1100 \\
\hline EBRG & 136 & $\begin{array}{l}\text { Venezuela: Edo. Aragua: Parque Nacional } \\
\text { Henri Pittier, Rancho Grande }\end{array}$ & Jun-76 & 259.8 & 1100 \\
\hline
\end{tabular}


APÉNDICE I (Continuación) / APENDIX 1 (Continued)

\begin{tabular}{|c|c|c|c|c|c|}
\hline Colección & $\begin{array}{c}\mathrm{N}^{\mathrm{o}} \\
\text { espécimen }\end{array}$ & Localidad & $\begin{array}{l}\text { Fecha de } \\
\text { colecta }\end{array}$ & $\begin{array}{c}\text { Precipitación } \\
\text { correspondiente al mes de } \\
\text { evento de captura registrada } \\
\text { en el PNHP }(\mathrm{mm})\end{array}$ & $\begin{array}{l}\text { Altitud } \\
\text { (metros) }\end{array}$ \\
\hline $\mathrm{EBRG}^{\dagger}$ & $\begin{array}{l}\text { 137-45 (nacidas } \\
\text { en cautiverio) }\end{array}$ & $\begin{array}{l}\text { Venezuela: Edo. Aragua: Parque Nacional } \\
\text { Henri Pittier, Rancho Grande }\end{array}$ & Jun-76 & 259.8 & 1100 \\
\hline EBRG & 146 & $\begin{array}{l}\text { Venezuela: Edo. Aragua: Parque Nacional } \\
\text { Henri Pittier, Rancho Grande }\end{array}$ & 1976 & Dato no precisado & 1100 \\
\hline EBRG & 147 & $\begin{array}{l}\text { Venezuela: Edo. Aragua: Parque Nacional } \\
\text { Henri Pittier, Rancho Grande }\end{array}$ & Mar-75 & 10 & 1100 \\
\hline EBRG & 148 & $\begin{array}{l}\text { Venezuela: Edo. Aragua: Parque Nacional } \\
\text { Henri Pittier, Rancho Grande }\end{array}$ & Mar-75 & 10 & 1100 \\
\hline EBRG & 149 & $\begin{array}{l}\text { Venezuela: Edo. Aragua: Parque Nacional } \\
\text { Henri Pittier, Rancho Grande }\end{array}$ & Abr-75 & 26.8 & 1100 \\
\hline EBRG & 150 & $\begin{array}{l}\text { Venezuela: Edo. Aragua: Parque Nacional } \\
\text { Henri Pittier, Rancho Grande }\end{array}$ & Abr-75 & 26.8 & 1100 \\
\hline EBRG & 151 & $\begin{array}{l}\text { Venezuela: Edo. Aragua: Parque Nacional } \\
\text { Henri Pittier, Rancho Grande }\end{array}$ & Mar-77 & 53 & 1100 \\
\hline EBRG & 152 & $\begin{array}{l}\text { Venezuela: Edo. Miranda: San José de } \\
\text { Los Altos }\end{array}$ & Dic-76 & & $\begin{array}{l}\text { Sin altitud; } \\
\quad 1350\end{array}$ \\
\hline EBRG & 1197 & $\begin{array}{l}\text { Venezuela: Edo. Aragua: Parque Nacional } \\
\text { Henri Pittier, Estación Biológica de } \\
\text { Rancho Grande, camino a la cumbre de } \\
\text { Rancho Grande }\end{array}$ & Mar-80 & 3.3 & 1200 \\
\hline EBRG & 3295 & $\begin{array}{l}\text { Venezuela: Dtto. Capital: Parque Nacional } \\
\text { Warairarepano (PN El Ávila), quebrada } \\
\text { Gamboa }\end{array}$ & Ene-94 & & Sin altitud \\
\hline EBRG & 3325 & $\begin{array}{l}\text { Venezuela: Edo. Yaracuy: Sierra de Aroa, } \\
\text { Los Bacos }\end{array}$ & Abr-96 & & 1600 \\
\hline EBRG & 3507 & $\begin{array}{l}\text { Venezuela: Dtto. Capital: Parque Nacional } \\
\text { Warairarepano (PN El Ávila), sector } \\
\text { Galipán }\end{array}$ & Abr-97 & & $\begin{array}{l}\text { Sin altitud; } \\
1500-1600\end{array}$ \\
\hline EBRG & 3508 & $\begin{array}{l}\text { Venezuela: Dtto. Capital: Parque Nacional } \\
\text { Warairarepano (PN El Ávila), sector } \\
\text { Galipán }\end{array}$ & Abr-97 & & $\begin{array}{l}\text { Sin altitud; } \\
1500-1600\end{array}$ \\
\hline EBRG & 3509 & $\begin{array}{l}\text { Venezuela: Dtto. Capital: Parque Nacional } \\
\text { Warairarepano (PN El Ávila), sector } \\
\text { Galipán }\end{array}$ & Abr-97 & & $\begin{array}{l}\text { Sin altitud; } \\
1500-1600\end{array}$ \\
\hline EBRG & 3510 & $\begin{array}{l}\text { Venezuela: Dtto. Capital: Parque Nacional } \\
\text { Warairarepano (PN El Ávila), sector } \\
\text { Galipán }\end{array}$ & Abr-97 & & $\begin{array}{l}\text { Sin altitud; } \\
1500-1600\end{array}$ \\
\hline EBRG & 4041 & $\begin{array}{l}\text { Venezuela: Edo. Miranda: San José de } \\
\text { Los Altos }\end{array}$ & Dic-81 & & 1350 \\
\hline EBRG & 4618 & $\begin{array}{l}\text { Venezuela: Edo. Falcón: Cerro Los } \\
\text { Caracoles }\end{array}$ & Sep-02 & & 1000 \\
\hline
\end{tabular}


APÉNDICE I (Continuación) / APENDIX 1 (Continued)

\begin{tabular}{|c|c|c|c|c|c|}
\hline Colección & $\begin{array}{c}\mathrm{N}^{\mathrm{o}} \\
\text { espécimen }\end{array}$ & Localidad & $\begin{array}{c}\text { Fecha de } \\
\text { colecta }\end{array}$ & $\begin{array}{c}\text { Precipitación } \\
\text { correspondiente al mes de } \\
\text { evento de captura registrada } \\
\text { en el PNHP }(\mathrm{mm})\end{array}$ & $\begin{array}{l}\text { Altitud } \\
\text { (metros) }\end{array}$ \\
\hline EBRG & 4619 & $\begin{array}{l}\text { Venezuela: Edo. Falcón: Cerro Los } \\
\text { Caracoles }\end{array}$ & Sep-02 & & 1000 \\
\hline $\mathrm{KU}$ & 125372 & Venezuela: Dtto. Capital, Caracas & May-39 & & Sin altitud \\
\hline KU & 125373 & Venezuela: Dtto. Capital, Caracas & May-39 & & Sin altitud \\
\hline KU & 133388 & $\begin{array}{l}\text { Venezuela: Edo. Aragua: Parque Nacional } \\
\text { Henri Pittier, en una quebrada ubicada } \\
\text { a } 0,5 \mathrm{Km} \text {. de la Estación Biológica de } \\
\text { Rancho Grande }\end{array}$ & Jun-70 & 393.1 & $\begin{array}{l}\text { Sin altitud; } \\
\quad 1040\end{array}$ \\
\hline $\mathrm{KU}$ & 133389 & $\begin{array}{l}\text { Venezuela: Edo. Aragua: Parque Nacional } \\
\text { Henri Pittier, en una quebrada ubicada } \\
\text { a } 0,5 \mathrm{Km} \text {. de la Estación Biológica de } \\
\text { Rancho Grande }\end{array}$ & Jun-70 & 393,1 & $\begin{array}{l}\text { Sin altitud; } \\
\quad 1040\end{array}$ \\
\hline KU & 166760 & $\begin{array}{l}\text { Venezuela: Edo. Aragua: Parque Nacional } \\
\text { Henri Pittier, Estación Biológica de } \\
\text { Rancho Grande }\end{array}$ & Ago-74 & 386,4 & $\begin{array}{l}\text { Sin altitud; } \\
\quad 1100\end{array}$ \\
\hline $\mathrm{KU}$ & 166761 & $\begin{array}{l}\text { Venezuela: Edo. Aragua: Parque Nacional } \\
\text { Henri Pittier, Estación Biológica de } \\
\text { Rancho Grande }\end{array}$ & Ago-74 & 386,4 & $\begin{array}{l}\text { Sin altitud; } \\
\quad 1100\end{array}$ \\
\hline $\mathrm{KU}$ & 166762 & $\begin{array}{l}\text { Venezuela: Edo. Aragua: Parque Nacional } \\
\text { Henri Pittier, Estación Biológica de } \\
\text { Rancho Grande }\end{array}$ & Ago-74 & 386,4 & $\begin{array}{l}\text { Sin altitud; } \\
\quad 1100\end{array}$ \\
\hline $\mathrm{KU}$ & 166763 & $\begin{array}{l}\text { Venezuela: Edo. Aragua: Parque Nacional } \\
\text { Henri Pittier, Estación Biológica de } \\
\text { Rancho Grande }\end{array}$ & Ago-74 & 386,4 & $\begin{array}{l}\text { Sin altitud; } \\
\quad 1100\end{array}$ \\
\hline KU & 166764 & $\begin{array}{l}\text { Venezuela: Edo. Aragua: Parque Nacional } \\
\text { Henri Pittier, Estación Biológica de } \\
\text { Rancho Grande }\end{array}$ & Ago-74 & 386,4 & $\begin{array}{l}\text { Sin altitud; } \\
\quad 1100\end{array}$ \\
\hline $\mathrm{KU}$ & 185732 & $\begin{array}{l}\text { Venezuela: Edo. Aragua: Parque Nacional } \\
\text { Henri Pittier, Estación Biológica de } \\
\text { Rancho Grande }\end{array}$ & Oct-74 & 369,4 & $\begin{array}{l}\text { Sin altitud; } \\
\quad 1100\end{array}$ \\
\hline KU & 185733 & $\begin{array}{l}\text { Venezuela: Edo. Aragua: Parque Nacional } \\
\text { Henri Pittier, Rancho Grande, Paso de } \\
\text { Portachuelo }\end{array}$ & Jun-75 & 100,7 & $\begin{array}{l}\text { Sin altitud; } \\
\quad 1100\end{array}$ \\
\hline $\mathrm{KU}$ & 185779 & $\begin{array}{l}\text { Venezuela: Edo. Aragua: Parque Nacional } \\
\text { Henri Pittier, Estación Biológica de } \\
\text { Rancho Grande }\end{array}$ & May-76 & & $\begin{array}{l}\text { Sin altitud; } \\
\quad 1100\end{array}$ \\
\hline KU & 185780 & $\begin{array}{l}\text { Venezuela: Edo. Aragua: Parque Nacional } \\
\text { Henri Pittier, Estación Biológica de } \\
\text { Rancho Grande }\end{array}$ & May-76 & 193,1 & $\begin{array}{l}\text { Sin altitud; } \\
\quad 1100\end{array}$ \\
\hline KU & 185781 & $\begin{array}{l}\text { Venezuela: Edo. Aragua: Parque Nacional } \\
\text { Henri Pittier, Estación Biológica de } \\
\text { Rancho Grande }\end{array}$ & Jun-76 & 259,8 & $\begin{array}{l}\text { Sin altitud; } \\
\quad 1100\end{array}$ \\
\hline
\end{tabular}


APÉNDICE I (Continuación) / APENDIX 1 (Continued)

\begin{tabular}{|c|c|c|c|c|c|}
\hline Colección & $\begin{array}{c}\mathrm{N}^{\circ} \\
\text { espécimen }\end{array}$ & Localidad & $\begin{array}{c}\text { Fecha de } \\
\text { colecta }\end{array}$ & $\begin{array}{c}\text { Precipitación } \\
\text { correspondiente al mes de } \\
\text { evento de captura registrada } \\
\text { en el PNHP }(\mathrm{mm})\end{array}$ & $\begin{array}{l}\text { Altitud } \\
\text { (metros) }\end{array}$ \\
\hline $\mathrm{KU}$ & 185782 & $\begin{array}{l}\text { Venezuela: Edo. Aragua: Parque Nacional } \\
\text { Henri Pittier, Estación Biológica de } \\
\text { Rancho Grande }\end{array}$ & Jun-76 & 259,8 & $\begin{array}{l}\text { Sin altitud; } \\
1.100\end{array}$ \\
\hline $\mathrm{KU}$ & 185785 & $\begin{array}{l}\text { Venezuela: Edo. Aragua: Parque Nacional } \\
\text { Henri Pittier, Estación Biológica de } \\
\text { Rancho Grande }\end{array}$ & Dic-76 & 24,9 & $\begin{array}{l}\text { Sin altitud; } \\
\quad 1100\end{array}$ \\
\hline $\mathrm{KU}$ & 209587 & $\begin{array}{l}\text { Venezuela: Edo. Aragua: Parque Nacional } \\
\text { Henri Pittier, Rancho Grande }\end{array}$ & 1946 & Dato no precisado & $\begin{array}{l}\text { Sin altitud; } \\
\quad 1100\end{array}$ \\
\hline $\mathrm{KU}$ & 209588 & $\begin{array}{l}\text { Venezuela: Edo. Aragua: Parque Nacional } \\
\text { Henri Pittier, Rancho Grande }\end{array}$ & 1946 & Dato no precisado & $\begin{array}{l}\text { Sin altitud; } \\
\quad 1100\end{array}$ \\
\hline $\mathrm{KU}$ & 209589 & $\begin{array}{l}\text { Venezuela: Edo. Aragua: Parque Nacional } \\
\text { Henri Pittier, Rancho Grande }\end{array}$ & 1946 & Dato no precisado & $\begin{array}{l}\text { Sin altitud; } \\
\quad 1100\end{array}$ \\
\hline $\mathrm{KU}$ & 209590 & $\begin{array}{l}\text { Venezuela: Edo. Aragua: Parque Nacional } \\
\text { Henri Pittier, Rancho Grande }\end{array}$ & 1946 & Dato no precisado & $\begin{array}{l}\text { Sin altitud; } \\
\quad 1100\end{array}$ \\
\hline MBUCV & 626 & $\begin{array}{l}\text { Venezuela: Edo. Aragua: Parque Nacional } \\
\text { Henri Pittier, entrada de la Estación } \\
\text { Biológica de Rancho Grande, Rancho } \\
\text { Grande }\end{array}$ & 1958 & Dato no precisado & 1100 \\
\hline MBUCV & 3261 & $\begin{array}{l}\text { Venezuela: Edo. Aragua: Parque Nacional } \\
\text { Henri Pittier, camino a La Toma, Rancho } \\
\text { Grande }\end{array}$ & 1955 & Dato no precisado & $\begin{array}{l}\text { Sin altitud; } \\
1120-1150\end{array}$ \\
\hline MBUCV & 1994 & $\begin{array}{l}\text { Venezuela: Edo. Aragua: Parque Nacional } \\
\text { Henri Pittier, Rancho Grande }\end{array}$ & 1951 & Dato no precisado & 1100 \\
\hline MBUCV & 6238 & $\begin{array}{l}\text { Venezuela: Edo. Aragua: Parque Nacional } \\
\text { Henri Pittier, Rancho Grande, Paso de } \\
\text { Portachuelo }\end{array}$ & 1971 & Dato no precisado & $\begin{array}{l}\text { Sin altitud; } \\
\quad 1225\end{array}$ \\
\hline MHNLS & 8095 & $\begin{array}{l}\text { Venezuela: Edo. Cojedes: Cerro Azul, Fila } \\
\text { La Blanquera }\end{array}$ & 1980 & & 1350 \\
\hline MHNLS & 5074 & $\begin{array}{l}\text { Venezuela: Edo. Aragua: El Guacatal, } \\
\text { Hacienda El Limón }\end{array}$ & 1970 & & 1400 \\
\hline MHNLS & 2942 & Venezuela: Edo. Delta Amacuro & 1950 & & Sin altitud \\
\hline MHNLS & 2954 & $\begin{array}{l}\text { Venezuela: Dtto. Capital: carretera vía El } \\
\text { Junquito-Colonia Tovar }\end{array}$ & 1967 & & Sin altitud \\
\hline MHNLS & 5540 & $\begin{array}{l}\text { Venezuela: Edo. Aragua: Parque Nacional } \\
\text { Henri Pittier, carretera Choroní }\end{array}$ & 1971 & Dato no precisado & 1640 \\
\hline MHNLS & 10000 & $\begin{array}{l}\text { Venezuela: Edo. Miranda: Altos de Pipe, } \\
\text { Instituto Venezolano de Investigaciones } \\
\text { Científicas (IVIC) }\end{array}$ & 1984 & & $\begin{array}{l}\text { Sin altitud; } \\
1500-1600\end{array}$ \\
\hline MHNLS & 4708 & $\begin{array}{l}\text { Venezuela: Edo. Aragua: El Guacatal, } \\
\text { Hacienda El Limón }\end{array}$ & 1969 & & 1400 \\
\hline
\end{tabular}


APÉNDICE I (Continuación) / APENDIX 1 (Continued)

\begin{tabular}{|c|c|c|c|c|c|}
\hline Colección & $\begin{array}{c}\mathrm{N}^{\circ} \\
\text { espécimen }\end{array}$ & Localidad & $\begin{array}{l}\text { Fecha de } \\
\text { colecta }\end{array}$ & $\begin{array}{c}\text { Precipitación } \\
\text { correspondiente al mes de } \\
\text { evento de captura registrada } \\
\text { en el PNHP }(\mathrm{mm})\end{array}$ & $\begin{array}{l}\text { Altitud } \\
\text { (metros) }\end{array}$ \\
\hline MHNLS & 4709 & $\begin{array}{l}\text { Venezuela: Edo. Aragua: El Guacatal, } \\
\text { Hacienda El Limón }\end{array}$ & 1969 & & 1400 \\
\hline MHNLS & 13290 & $\begin{array}{l}\text { Venezuela: Dtto. Capital: Parque Nacional } \\
\text { Warairarepano (PN El Ávila) Boca del } \\
\text { Tigre, Fila Maestra }\end{array}$ & 1994 & & 1800 \\
\hline MHNLS & 13544 & $\begin{array}{l}\text { Venezuela: Edo. Miranda: sector El } \\
\text { Amarillo, San Antonio de los Altos }\end{array}$ & 1996 & & $\begin{array}{l}\text { Sin altitud; } \\
1300-1350\end{array}$ \\
\hline MHNLS & 8599 & $\begin{array}{l}\text { Venezuela: Edo. Aragua: Parque Nacional } \\
\text { Henri Pittier, Estación Biológica de } \\
\text { Rancho Grande, Rancho Grande }\end{array}$ & 1974 & Dato no precisado & $\begin{array}{l}\text { Sin altitud; } \\
\quad 1100\end{array}$ \\
\hline MHNLS & 11676 & $\begin{array}{l}\text { Venezuela: Edo. Yaracuy: Finca El Jaguar, } \\
\text { Sierra de Aroa }\end{array}$ & 1987 & & $\begin{array}{l}\text { Sin altitud; } \\
1500-1600\end{array}$ \\
\hline MHNLS & 2007 & $\begin{array}{l}\text { Venezuela: Dtto. Capital: Parque Nacional } \\
\text { Warairarepano (PN El Ávila), norte de } \\
\text { Caracas }\end{array}$ & 1966 & & Sin Altitud \\
\hline MHNLS & 1199 & Venezuela: Dtto. Capital: El Junquito & 1955 & & 1200 \\
\hline MHNLS & 1309 & $\begin{array}{l}\text { Venezuela: Edo. Aragua: Parque Nacional } \\
\text { Henri Pittier, Rancho Grande }\end{array}$ & 1956 & Dato no precisado & 1100 \\
\hline MHNLS & 3701 & $\begin{array}{l}\text { Venezuela: Edo. Aragua: El Guacatal, } \\
\text { Hacienda El Limón }\end{array}$ & 1966 & & 1400 \\
\hline MHNLS & 3695 & $\begin{array}{l}\text { Venezuela: Edo. Aragua: El Guacatal, } \\
\text { Hacienda El Limón }\end{array}$ & 1968 & & 1400 \\
\hline MHNLS & 3696 & $\begin{array}{l}\text { Venezuela: Edo. Aragua: El Guacatal, } \\
\text { Hacienda El Limón }\end{array}$ & 1968 & & 1400 \\
\hline MHNLS & 3697 & $\begin{array}{l}\text { Venezuela: Edo. Aragua: El Guacatal, } \\
\text { Hacienda El Limón }\end{array}$ & 1968 & & 1400 \\
\hline MHNLS & 3698 & $\begin{array}{l}\text { Venezuela: Edo. Aragua: El Guacatal, } \\
\text { Hacienda El Limón }\end{array}$ & 1968 & & 1400 \\
\hline MIZA & 500 & $\begin{array}{l}\text { Venezuela: Edo. Aragua: Parque Nacional } \\
\text { Henri Pittier, Rancho Grande, Sendero } \\
\text { Andrew Field }\end{array}$ & Feb-94 & 11 & 1115 \\
\hline MIZA & 594 & $\begin{array}{l}\text { Venezuela: Edo. Aragua: Parque Nacional } \\
\text { Henri Pittier, Estación Biológica de } \\
\text { Rancho Grande, Rancho Grande }\end{array}$ & Feb-79 & 4,6 & 1100 \\
\hline MIZA & 595 & $\begin{array}{l}\text { Venezuela: Edo. Aragua: Parque Nacional } \\
\text { Henri Pittier, Estación Biológica de } \\
\text { Rancho Grande, Rancho Grande }\end{array}$ & May-79 & 188,9 & 1100 \\
\hline MIZA & 596 & $\begin{array}{l}\text { Venezuela: Edo. Aragua: Parque Nacional } \\
\text { Henri Pittier, Rancho Grande, Paso de } \\
\text { Portachuelo }\end{array}$ & Oct-79 & 114,7 & 1125 \\
\hline
\end{tabular}


APÉNDICE I (Continuación) / APENDIX 1 (Continued)

\begin{tabular}{|c|c|c|c|c|c|}
\hline Colección & $\begin{array}{c}\mathrm{N}^{\mathrm{o}} \\
\text { espécimen }\end{array}$ & Localidad & $\begin{array}{l}\text { Fecha de } \\
\text { colecta }\end{array}$ & $\begin{array}{c}\text { Precipitación } \\
\text { correspondiente al mes de } \\
\text { evento de captura registrada } \\
\text { en el PNHP }(\mathrm{mm})\end{array}$ & $\begin{array}{l}\text { Altitud } \\
\text { (metros) }\end{array}$ \\
\hline MIZA & 597 & $\begin{array}{l}\text { Venezuela: Edo. Aragua: Parque Nacional } \\
\text { Henri Pittier, Rancho Grande, Paso de } \\
\text { Portachuelo }\end{array}$ & May-80 & 253,5 & 1125 \\
\hline MIZA & 674 & $\begin{array}{l}\text { Venezuela: Edo. Aragua: Parque Nacional } \\
\text { Henri Pittier, Estación Biológica de } \\
\text { Rancho Grande, Rancho Grande }\end{array}$ & Jun-85 & 303,4 & 1100 \\
\hline MIZA & 726 & $\begin{array}{l}\text { Venezuela: Edo. Aragua: Parque Nacional } \\
\text { Henri Pittier, Estación Biológica de } \\
\text { Rancho Grande, Rancho Grande }\end{array}$ & Jun-85 & 303,4 & 1.100 \\
\hline MIZA & 731 & $\begin{array}{l}\text { Venezuela: Edo. Aragua: Parque Nacional } \\
\text { Henri Pittier, Rancho Grande, Paso de } \\
\text { Portachuelo-Cerro Periquito }\end{array}$ & Ene-87 & 11,3 & 1200 \\
\hline MIZA & 824 & $\begin{array}{l}\text { Venezuela: Edo. Aragua: Parque Nacional } \\
\text { Henri Pittier, Quebrada Guacamaya }\end{array}$ & Feb-96 & 18 & 890 \\
\hline MIZA & 825 & $\begin{array}{l}\text { Venezuela: Edo. Aragua: Parque Nacional } \\
\text { Henri Pittier, Quebrada Guacamaya }\end{array}$ & Feb-96 & 18 & 890 \\
\hline MIZA & 826 & $\begin{array}{l}\text { Venezuela: Edo. Aragua: Parque Nacional } \\
\text { Henri Pittier, Quebrada Guacamaya }\end{array}$ & Feb-96 & 18 & 890 \\
\hline MIZA & 874 & Venezuela: Edo. Carabobo: Cerro La Copa & Sep-07 & & 1450 \\
\hline MIZA & 876 & Venezuela: Edo. Carabobo: Cerro La Copa & Sep-07 & & 1450 \\
\hline UIMNH & 61870 & $\begin{array}{l}\text { Venezuela: Edo. Aragua: Parque Nacional } \\
\text { Henri Pittier, Rancho Grande }\end{array}$ & 1939 & Dato no precisado & $\begin{array}{l}\text { Sin altitud; } \\
\quad 1100\end{array}$ \\
\hline USNM & 121164 & Venezuela: Edo. Aragua: Colonia Tovar & 1929 & & 1800 \\
\hline USNM & 121165 & Venezuela: Edo. Aragua: Colonia Tovar & 1929 & & 1800 \\
\hline USNM & 121166 & Venezuela: Edo. Aragua: Colonia Tovar & 1929 & & 1800 \\
\hline USNM & 216642 & $\begin{array}{l}\text { Venezuela: Dtto. Capital: Parque Nacional } \\
\text { Macarao }\end{array}$ & 1965 & & 2060 \\
\hline TWC & 59036 & $\begin{array}{l}\text { Venezuela: Edo. Aragua: Parque Nacional } \\
\text { Henri Pittier, Rancho Grande, Cerro } \\
\text { Periquito }\end{array}$ & 1980 & Dato no precisado & 1350 \\
\hline TWC & 59037 & $\begin{array}{l}\text { Venezuela: Edo. Aragua: Parque Nacional } \\
\text { Henri Pittier, Rancho Grande, Cerro } \\
\text { Periquito. }\end{array}$ & 1980 & Dato no precisado & 1350 \\
\hline ZSM & ZSM 67/1937 & $\begin{array}{l}\text { Venezuela: Dtto. Capital: Hacienda Los } \\
\text { Venados, Parque Nacional Warairarepano } \\
\text { (P. N. El Ávila) }\end{array}$ & 1937 & & $\begin{array}{l}\text { Sin altitud; } \\
\quad 1550\end{array}$ \\
\hline
\end{tabular}

